\title{
AUDIOVISUAL PARTICIPATIVO: CONVERGENCIA ENTRE COMUNICACIÓN Y MEDIO AMBIENTE
}

\author{
Angie Ramírez RuedA* \\ Universidad Pontificia Bolivariana, Colombia \\ anguieramirez80@gmail.com \\ Blanca Aracely López Rueda** \\ Universidad Pontificia Bolivariana, Colombia \\ aracely.lopez@upb.edu.co
}

Recibido: 12/5/2019/ Aceptado: 11/3/2020

Doi: $10.26439 /$ contratexto2020.n033.4789

Resumen. Este artículo presenta diversas reflexiones sobre el uso del audiovisual participativo como una herramienta que moviliza a las comunidades hacia otras formas de comunicación, donde se valora el trabajo colaborativo, con el fin de que sean ellas las creadoras de sus propios contenidos, y así se repiensen sus territorios. Teniendo como punto de partida esta premisa, se formuló la estrategia educomunicativa "Pescando historias. Memorias de un llanitero", desarrollada con adolescentes y jóvenes del corregimiento El Llanito, en Barrancabermeja, Colombia, quienes se formaron en producción audiovisual con énfasis en medio ambiente. La metodología implementada fue del orden cualitativo, desde la línea de la investigación acción (IA), para propiciar espacios de participación y diálogo con los jóvenes. Esta investigación se apoyó en la educomunicación durante la formación pedagógica desde una perspectiva crítica para fortalecer los discursos y prácticas medioambientales de los participantes, al tiempo que se capacitaban en el uso de esta herramienta comunicativa.

Palabras clave: audiovisual participativo / jóvenes / recursos naturales / educomunicación / TIC

\footnotetext{
* Estudiante de Comunicación Social-Periodismo por la Universidad Pontificia Bolivariana, Colombia (https:// orcid.org/0000-0002-8792-0429).

** Periodista por la Universidad Pontificia Bolivariana, Colombia (https://orcid.org/0000-0003-4123-8616).
} 


\section{PARTICIPATORY AUDIOVISUALS: A CONVERGENCE BETWEEN COMMUNICATION AND ENVIRONMENT}

Abstract. This article presents various reflections on the use of participatory audiovisuals as a tool that mobilizes communities towards other forms of communication, where collaborative work is valued so that such communities are the creators of their own content, and thus rethink their territories. Taking this premise as a starting point, the educommunicative strategy "Pescando Historias. Memorias de un Llanitero" (Catching Stories. Memories of a Llanitero) was developed with adolescents and young people from El Llanito village in Barrancabermeja, Colombia, who were trained in audiovisual productions with emphasis in environment. The research used a qualitative methodology based on Action Research (IA), thus providing spaces for participation and dialogue with the young people. From a critical perspective, this research was based on educommunication during the pedagogical training to strengthen the discourses and environmental practices of the participants who, at the same time, were trained in the use of this communicative tool.

Keywords: participatory audiovisuals / young people / natural resources / educommunication / TICS

\section{AUDIOVISUAL PARTICIPATIVO: CONVERGENTE ENTRE COMUNICAÇ̃̃O E MEIO AMBIENTE}

Resumo. Este artigo apresenta várias reflexões sobre o uso dos audiovisuais participativos como ferramenta que mobiliza as comunidades para outras formas de comunicação, onde o trabalho colaborativo é valorizado para que elas sejam criadoras de seus próprios conteúdos e, assim, repensem seus territórios. Tendo como ponto de partida esta premissa, a estratégia educomunicativa "Pescando historias. Memorias de un llanitero", foi desenvolvido com adolescentes e jovens do Corregimiento el Llanito em Barrancabermeja, Colômbia, que foram treinados em produção audiovisual com ênfase no meio ambiente. A metodologia implementada foi qualitativa, a partir da linha de pesquisa em ação (Al), proporcionando espaços de participação e diálogo com os jovens. Esta pesquisa foi baseada na educomunicação durante a formação pedagógica a partir de uma perspectiva crítica para fortalecer os discursos e práticas ambientais dos participantes, ao mesmo tempo em que eles foram treinados no uso dessa ferramenta comunicativa.

Palavras-chave: audiovisual participativo / jovem / recursos naturais / educommunication / TIC 


\section{INTRODUCCIÓN}

En el mundo existe gran preocupación por la conservación de la fauna, la flora y los recursos naturales que a diario se ven amenazados por la industrialización y el afán de consumo por parte de los seres humanos; sin embargo, esta cuestión no termina ahí, puesto que la falta de conciencia y la indiferencia de la sociedad hacia esta situación es aún mayor. Por tal razón, la presencia de los líderes ambientales ha sido de gran importancia para la defensa y protección de los diversos ecosistemas, pero ejercer esta labor implica un riesgo para la vida y la tranquilidad de aquellas personas, ya que sus acciones y esfuerzos representan para otros una amenaza.

En el caso colombiano, el país ha vivido por más de cincuenta años una guerra interna que ha cobrado la vida de miles de personas inocentes, entre las cuales se encuentran aquellos actores sociales que han alzado sus voces para denunciar abusos por parte de grupos armados ilegales que se aprovechan de la ausencia del Estado para ejercer un control de dominio en ciertos territorios y cometer crímenes atroces. Ante este panorama, los líderes ambientales fueron tomando la iniciativa de ser los voceros de sus comunidades para visibilizar esas acciones que han afectado de forma negativa los ecosistemas. De esa manera, ser defensor se convirtió en una de las labores más peligrosas y, en la mayoría de las veces, en un trabajo filantrópico que solo busca el bienestar de los habitantes.

A pesar del flagelo que se vive, muchos ciudadanos no han abandonado el liderazgo que tienen en sus comunidades, especialmente en la defensa del medio ambiente, pues como se mencionó anteriormente es una de las principales problemáticas que enfrenta el mundo en la actualidad. Sin embargo, algunos de esos líderes ambientales han sido silenciados, tal como ocurrió con Luis Alberto Arango, conocido también como Lucho Arango, quien se dedicó a la defensa de la pesca artesanal en el corregimiento El Llanito, Barrancabermeja, Colombia, para preservar las especies de la ciénaga que día a día estaban siendo amenazadas por diferentes factores externos, lo que generaba un daño para la sostenibilidad de este recurso en el territorio.

El liderazgo efectuado por Lucho Arango en la ciénaga El Llanito dejó en evidencia la falta de regulación por parte del Estado para cuidar este tipo de recursos, pues las leyes para prohibir herramientas como el trasmallo no son del todo contundentes; además, denunció la actuación de ganaderos y palmicultores, quienes utilizaban los terrenos aledaños a la ciénaga para llevar a cabo sus actividades económicas, las cuales aumentaban la sedimentación del complejo cenagoso. Por otra parte, y sin que sean menos importantes, están los derrames de petróleo que en varias ocasiones han causado la mortalidad de cientos de especies. Ante tal situación, Lucho y otros pescadores unieron fuerzas para defender lo que los representaba como llaniteros, pero no fueron escuchados y, en el 2009, el líder terminó asesinado por la banda criminal Los Rastrojos, grupo ilegal que se conformó tras la desmovilización de los paramilitares en la región. 


\section{CONTEXTO TERRITORIAL DEL MAGDALENA MEDIO}

El Magdalena Medio, donde se ubica Barrancabermeja, es una de las regiones más importantes de Colombia, pero a su vez una de las zonas con mayor violencia debido a la poca presencia del Estado, donde tienen gran actividad los grupos armados ilegales ya mencionados. Además, es considerada geográficamente como un punto estratégico de comunicación, pues conecta al Caribe con el interior del país. También es una fuente de grandes riquezas naturales, pues está formada por el río Magdalena, que alberga infinitas especies de animales y vegetales, así como recursos minerales; por ello, en Barrancabermeja se encuentra la refinería de Ecopetrol, al igual que empresas del sector agropecuario e industrial que han venido desarrollando sus actividades de explotación.

La dinámica de violencia en el territorio facilitó el desplazamiento forzado de muchas familias que a causa de las amenazas decidieron abandonar sus propiedades, lo que ocasionó, por ejemplo, la expansión de grandes plantaciones de palma africana por parte de terratenientes que se apoderaron de esos terrenos:

\footnotetext{
Las empresas palmicultoras, aprovechando las desterritorializaciones masivas y las masacres cometidas por grupos paramilitares, especialmente en el Magdalena Medio, el Chocó y el nororiente colombiano, se han asentado allí para sembrar miles de hectáreas de palma africana [...]. Como se ve, estos enclaves extractivos se ubicaron en zonas históricamente conflictivas y lograron estar allí violando los derechos humanos de los habitantes de esos lugares para favorecer intereses concretos. (Díaz, 2017, p. 68)
}

Aunado a lo anterior, la implementación de megaproyectos como la Ruta del Sol, la Ruta del Cacao y la Hidroeléctrica de Sogamoso han traído serias consecuencias sociales, económicas y ambientales en la región. Así, por ejemplo, la última obra influyó en el nivel del río Sogamoso, que abastece a la ciénaga El Llanito, y por esta razón los pescadores del corregimiento que lleva el mismo nombre vieron afectados los ciclos de vida y desarrollo de los peces, los cuales repercuten directamente en los tiempos de pesca. Para este sector poblacional, la falta de apoyo gubernamental, el uso del trasmallo y la poca renovación generacional condujeron a que, diez años después del asesinato de Lucho Arango, se formulara una estrategia educomunicativa que, a través de la conformación de un colectivo de comunicación con adolescentes y jóvenes, promoviera la pesca artesanal como una forma amable de explotación de los recursos naturales.

\section{COMUNICACIÓN Y AUDIOVISUAL PARTICIPATIVO}

La comunicación, concebida desde el paradigma de la educomunicación, permite establecer escenarios comunitarios que a través del diálogo logren la participación activa y democrática de las personas, acercándolas a entender y comprender sus realidades, pues es "la forma de percibir el mundo, de percibirse a uno mismo en él, como un 
conjunto de valores, creencias e ideas que están en constante intercambio" (Ceballos, 1999, p. 102). Esto lleva a dotar de sentido los procesos comunicativos que al final conducirán a cambiar y mejorar las cotidianidades.

Aunque para Freire (1969) los medios masivos son los que han ido moldeando los valores y gustos, también señala que, vistos desde otra perspectiva, permiten rescatar las tradiciones e identidades de una comunidad; por ello, el pedagogo brasileño acerca el concepto de educomunicación a una estrategia de formar personas críticas, autónomas y conscientes. La comunicación forja una interacción con los otros, fomentando el diálogo y los conocimientos, porque está basada en un "modelo horizontal", donde no hay espacio para lo burocrático ni para la imposición de saberes, pues todos pueden asumir el rol de interlocutores y propiciar una red social de interacciones.

Así pues, la educomunicación entrelaza los saberes con el hacer, con lo cual motiva la imaginación y creatividad de los individuos para poner en práctica lo adquirido, y se apoya en los medios alternativos no solo como herramientas para comunicar, sino que a su vez formen actitudes por medio de nuevos comportamientos. De esta manera, apuesta por la formación de sujetos sociales que se preocupen por las necesidades de su entorno y cuyo accionar impulse al resto de la comunidad a movilizarse en la creación de contenidos que lleven a establecer espacios de reflexión. En este sentido, se convierte en la "opción que surge de y para la comunidad, se origina en las mentes de los individuos que, inspirados en la realidad, proponen una mirada ajena a la del poder, que por lo regular resulta más crítica que la difundida por los medios tradicionales y controlados" (Corrales y Hernández, 2009, p. 4). De tal modo, el proceso será más participativo y ellos mismos podrán discernir las soluciones y lograr el empoderamiento.

La estrategia "Pescando historias. Memorias de un llanitero" buscó que los adolescentes y jóvenes se apropiaran de los saberes y redescubrieran la importancia de la práctica de la pesca artesanal para el cuidado de la ciénaga El Llanito, al igual que para la conservación de los recursos naturales de su territorio. Los participantes acudieron a la producción del audiovisual participativo como el medio para comunicar, pues, como White lo define, es "una herramienta para el desarrollo grupal y comunitario [...]. Genera una conciencia crítica que actúa como base para la creatividad y la comunicación. Por lo tanto, tiene el potencial para provocar cambios personales, sociales, políticos y culturales" (citado por Rocha, 2018, p. 52).

En el pasado era difícil pensar que una comunidad se organizara para producir un audiovisual, pues los equipos necesarios no eran asequibles por su elevado costo, su uso requería de un proceso formativo y el acceso a la información también representaba un obstáculo. Sin embargo, los avances tecnológicos han permitido reducir esta brecha y varias experiencias se han gestado en la creación del video participativo, que propicia espacios de diálogo democrático (Mosangini, 2010). 
La utilización del audiovisual participativo en experiencias comunitarias se remonta a 1967, específicamente, en una comunidad pesquera perteneciente al sur de Canadá. Esta iniciativa fue impulsada por Don Snowden, quien logró que los isleños visibilizaran sus problemáticas y, a partir de ello, se organizaran para proponer soluciones que beneficiaran a todos (Lunch, 2006). Posteriormente, compartieron los videos con algunos representantes del gobierno; esto permitió dar a conocer las necesidades que pasaban en su territorio para gestionar las ayudas que requerían. Lo anterior es un ejemplo de cómo a través del video participativo se pueden abrir caminos para la transformación social, pues

Hacer video participativo es una manera pedagógica de comprender nuestra responsabilidad como actores sociales dentro de nuestras propias comunidades; es un modo de hacer valer nuestra propia voz ante quienes deciden no escucharnos, incluso si aquellos sordos, distraídos, somos nosotros mismos. (Espinosa, 2012, p. 74)

Ahora bien, la generación del producto no es el fin del audiovisual participativo, pues el video es un medio con el que se puede vislumbrar la realidad que está a nuestro alrededor, pero es aún más significativo el proceso que puede llevar a la transformación. Es entonces cuando la comunidad le da importancia al trabajo en equipo y un valor a los saberes que tienen los demás, porque estos a su vez son compartidos con todos (Mosangini, 2010). Tal como se evidencia en la experiencia "Poniendo las manos sobre los medios", impulsada por el Centro de Creación de Imagen Popular (CECIP), en Brasil, quienes en colaboración con la comunidad crearon programas televisivos que eran transmitidos en lugares públicos, y estos, a su vez, generaron espacios de debate y discusión frente al papel de la comunicación. Debido al gran impacto de esta estrategia, se implementó en otros escenarios, como la escuela, razón por la cual "fue evidente para los maestros la visión de cuánto los medios, la televisión y el video, en especial, pueden tornarse en una poderosa herramienta pedagógica" (Ceccon, 2006, p. 257).

\section{ADOLESCENTES Y JÓVENES FRENTE AL USO DEL AUDIOVISUAL PARTICIPATIVO}

La participación de los adolescentes y jóvenes en los diferentes escenarios que a diario se viven en el mundo ha ido aumentando. Muchos de ellos han dirigido su accionar a la formación o participación en colectivos de comunicación que impulsan espacios para el arte, la cultura y el medio ambiente, tal como se puede observar en el proyecto "Manglar vivo", desarrollado en dos comunidades costeras del sur de Cuba, Artemisa y Mayabeque; sus participantes acudieron a la producción del audiovisual participativo, con el cual aprendieron la importancia del proceso, porque con este "no solo se resaltaron las posibilidades para la sensibilización y movilización social, también permite expresar creencias, maneras de pensar, e ideas" (Menéndez y Pedroso, 2017, p. 159). Así 
se convirtió en el medio más idóneo para comunicar las problemáticas ambientales que ellos mismos identificaron en sus territorios, al igual que posibles soluciones con el fin de mitigar los daños.

Por esta razón, "Pescando historias. Memorias de un llanitero" buscó que los mismos jóvenes fortalecieran su participación en la comunidad, que pudieran reconocer la importancia del cuidado de la ciénaga y de los recursos naturales de su territorio. Esta iniciativa es similar a la adelantada en Veracruz, México, donde se llevó a cabo la experiencia "El documental participativo como proceso educativo: hacia la realización de proyectos audiovisuales con enfoque intercultural", centrado en rescatar los saberes ancestrales como la pesca tradicional, que estaba limitada al pequeño grupo poblacional que la practicaba y que, además, estaba "siendo impactada de manera negativa por la contaminación industrial procedente de la rama petroquímica y por los drenajes urbanos que en algunos casos se vierten de manera directa a los cuerpos de agua" (Sandoval, 2015, p. 112).

Cerca de diez adolescentes se organizaron para realizar un documental de enfoque intercultural, donde narraban aquellas particularidades que forman parte de sus tradiciones e identidades, al tiempo que fortalecían sus habilidades comunicativas, reflexivas e investigativas. La relación que existe entre esta iniciativa, la de "Manglar vivo", y lo que se buscó en el Llanito es que ambos proyectos apuntan a ser un primer acercamiento de la comunidad a su territorio, donde esta pueda apropiarse tanto de los conocimientos como de las herramientas; así las personas harán uso de los procesos de diálogo y reflexión que más adelante podrían llevarlas a acciones comunitarias que se gesten desde la propia iniciativa del grupo y respalden el bienestar de todos. Al respecto, Sandoval (2015) afirma "[...] que los actores involucrados en la experiencia siguen desarrollando planes para la realización de nuevas producciones audiovisuales en la comunidad” (p. 112).

Otra investigación que se enmarca en la dinámica del video colaborativo es "El audiovisual participativo y las ciudadanías de alta intensidad en Cazuca (Colombia)" (Rocha, 2018), que estuvo encaminada hacia la identificación de conflictos que los mismos habitantes percibían en sus comunidades. La estrategia se implementó con jóvenes provenientes de Soacha y Cundinamarca, basándose en la realización de talleres formativos con el objetivo de caracterizar las problemáticas que, en este caso, fueron de tipo social y ambiental, "como el deterioro de los humedales y los recursos hídricos". Posteriormente, pasaron a la generación y definición de ideas por parte de los actores del proyecto a fin de organizar el plan de rodaje y hacer las grabaciones. Por último, llegaron a la etapa de socialización con los demás. Con lo anterior se evidencia que el uso de estas narrativas es adaptable a cualquier escenario mientras exista el compromiso y la disposición de los participantes. Así pues, "se buscó que estos actores sociales reflexionaran [...] utilizando el video como herramienta de visualización y participación" (Rocha, 2018, p. 48). 


\section{METODOLOGÍA}

Pasados ya diez años de la muerte de Lucho Arango, líder ambiental que visibilizó en su comunidad la importancia de la ciénaga para su territorio, nace el proyecto "Pescando historias. Memorias de un llanitero", que implementó una estrategia educomunicativa dirigida a adolescentes y jóvenes para que de forma colaborativa redescubrieran su territorio por medio de la fotografía, recuperaran las tradiciones orales de los pescadores con el uso de la radio y, a través del audiovisual participativo, pudieran elaborar diferentes narrativas que reflejaran la importancia del cuidado de la ciénaga El Llanito y los recursos naturales. De esta manera, propicia espacios para la reflexión grupal sobre el impacto que se genera en el entorno cuando no hay un adecuado manejo ambiental.

En ese sentido, la investigación se desarrolló con el método cualitativo, que centra su estudio en el sujeto con el fin de conocer sus valoraciones y apreciaciones sobre sus actuaciones y las de su comunidad, y que forman parte de la cotidianidad. Este enfoque es característico de la investigación social donde prevalecen el saber y la participación comunitaria. Esta última es, para los investigadores, el momento más relevante de las intervenciones porque es entonces cuando se crean lazos de confianza entre las partes. Según Galeano (2004), es importante que "privilegian lo local, lo cotidiano y lo cultural para comprender la lógica y el significado que tienen los procesos sociales para los propios actores, que son quienes viven y producen la realidad sociocultural" (p. 21).

Asimismo, la línea de trabajo que respalda este proyecto es la investigación acción (IA) dirigida a la transformación social en colaboración con la comunidad, pues al mismo tiempo que se indaga se va interviniendo, ya que los datos obtenidos funcionan como guía para la toma de decisiones. Stringer (citado por Sampieri, 2014) concibe a la IA como democrática, porque prevalece el trabajo mancomunado entre facilitadores y participantes; también es equitativa, puesto que cualquier contribución es bien aceptada, pero a su vez puede ser detonadora en busca de mejorar las condiciones de vida. Esto implica llevar a cabo acciones concretas y, para que se logren, es necesario que sea liberadora porque su fin es combatir la injusticia social.

Lewin (citado por Mejía, Novoa, Ñaupas y Villagómez, 2014) menciona cuatro pasos para llevar a cabo la investigación acción. El primero es la planificación, que para esta investigación se organizó en dos etapas: la aplicación de un diagnóstico participativo con adolescentes y jóvenes del corregimiento El Llanito, Barrancabermeja, que utilizó las técnicas de la entrevista semiestructurada y de grupos focales que sirvieron para conocer los imaginarios que tenían acerca de la pesca artesanal como una forma amable de explotación de los recursos naturales en la ciénaga; y el diseño de los talleres formativos desde los tres ejes por trabajar: la fotografía, la radio y el audiovisual participativo.

En segundo lugar, se identificó el actuar, que correspondió al trabajo colaborativo por parte de los participantes, quienes se apropiaron de las herramientas para redescubrir 
su territorio, recuperar la tradición oral y reflexionar sobre el cuidado y preservación de los recursos naturales. En tercer lugar, se acudió a la socialización a través de un espacio que involucró tanto a los estudiantes como a los docentes y padres de familia con el objetivo de dar a conocer los contenidos elaborados en cada uno de los talleres. Finalmente, la última etapa que señala Lewis consiste en propiciar la reflexión grupal frente a los temas trabajados por parte del colectivo.

Los participantes de la estrategia educomunicativa "Pescando historias. Memorias de un llanitero" fueron adolescentes y jóvenes entre 12 y 17 años, pertenecientes a la institución educativa San Marcos del corregimiento El Llanito. En total se focalizó un grupo de treinta estudiantes con grados de escolaridad que iban desde quinto primaria hasta undécimo grado de bachillerato. Todos ellos eran habitantes del corregimiento cuyos lugares de residencia se distribuían entre el casco urbano y sus veredas.

El diseño del taller formativo del audiovisual participativo se organizó en tres momentos. El primero fue sensibilizar a los participantes frente a la herramienta; esto se logró por medio de una dramatización a cargo de los facilitadores, donde se presentó a través de una historia la importancia de cuidar el medio ambiente y las consecuencias del manejo irresponsable de los residuos sólidos. Así los jóvenes tuvieron un acercamiento con la estructura del guion, sin tener que abordarlo primero desde la teoría; más bien, lo que se buscó fue socializar su estructura en el marco de un ejercicio práctico y que ellos no lo vieran como un aspecto complejo.

Además, la actividad de sensibilización permitió enlazar el discurso teórico con el lenguaje audiovisual, que fue la segunda parte del taller. A partir de la dramatización, el facilitador, en colaboración con los participantes, fueron comprendiendo los conceptos del audiovisual participativo: las etapas que implican su elaboración, es decir, preproducción, producción y posproducción; también se presentaron productos audiovisuales elaborados en otras experiencias para reforzar la explicación de las fases de producción y las responsabilidades que implica cada uno de los roles.

Por último, estuvo el momento de creación. En esta etapa los treinta jóvenes se organizaron en tres grupos de trabajo. Para definir el tema de sus audiovisuales, se implementó una lluvia de ideas con el fin de que los participantes identificaran las problemáticas medioambientales que enfrenta su territorio, lo que facilitó la construcción narrativa del video; así cada equipo realizó la estructuración del guion y la organización de las locaciones donde iban a llevar a cabo las grabaciones. Durante la realización del trabajo de campo, se hizo retroalimentación en los grupos y se tomaron decisiones frente al uso de planos, cambios en los diálogos y participación de personas de la comunidad. Esto facilitó en los estudiantes la comprensión del proceso y generó la necesidad de adaptarse a las dinámicas propias de la creación de contenido audiovisual, que no siempre se dan tal cual como se planean y es durante las grabaciones cuando se pueden 
enriquecer los mensajes con la inclusión de elementos que surgen directamente en la acción. De alguna forma, se trata de poner a prueba la capacidad de dejarse sorprender y de aprovechar los nuevos recursos que se encuentran inmersos en el contexto.

\section{RESULTADOS}

Ahora bien, en el marco de los procesos educomunicativos prevalece la construcción de espacios dialógicos que permiten la participación de las comunidades. Este modelo de aprendizaje fortalece las competencias comunicativas y logra que los propios actores sociales repiensen críticamente sus cotidianidades para que, posteriormente, reflexionen sobre sus acciones. En esta investigación, el audiovisual participativo fue el pretexto para que los adolescentes y jóvenes reflexionaran a profundidad sobre el cuidado de la ciénaga y la protección de los recursos naturales de su territorio. Finalmente, se obtuvieron tres productos que fueron analizados desde una matriz con el objetivo de establecer sus relaciones y diferencias.

En primera instancia, se examinaron los aspectos más relevantes de cada una de las etapas (preproducción, producción y posproducción) que fueron significativas para el proceso participativo. Al momento de escribir el guion, en la planificación del storyboard y en la definición de los roles en cada uno de los grupos, se evidenció que una gran parte de los participantes no tenía afinidad con la etapa de preproducción, la cual implica un mayor esfuerzo en la estructuración de ideas y recomendaciones para el momento de realizar las grabaciones; sin embargo, los jóvenes lograron organizarse para llevar a cabo la realización del audiovisual.

En la etapa de producción, al salir a campo, los jóvenes lograron entender cada una de las responsabilidades que implicaba el rol que estaban desempeñando, así como la importancia de liderar al grupo y que este, a su vez, escuchara las indicaciones para llevar a cabo las grabaciones. Cada uno de los grupos de trabajo vivenció algunas situaciones que se presentaron en esta fase como, por ejemplo, tener que prestar atención a elementos distractores que pudiesen afectar el desarrollo de las escenas, tomar una postura crítica para repetir las grabaciones las veces que fuese necesario, comprender que plasmar las ideas en la realidad requiere de más esfuerzo, al igual que enfrentarse a la poca colaboración de la comunidad cuando buscaban conocer sus opiniones sobre el cuidado medioambiental, puesto que, al ser observados con una herramienta como la cámara de video, estos perdían su disposición para ser entrevistados.

En cuanto a la posproducción, no se tuvieron en cuenta para el análisis ítems relacionados con la calidad de la imagen, ya que los participantes no contaban con la experticia técnica en cuanto a la producción, proceso que fue realizado originalmente por ellos. Sin embargo, se resalta la buena ejecución de los planos, ángulos y encuadres de cada una de las tomas, aspecto que tuvieron muy presente con el fin de mantener la estética 
que buscaban capturar, dado que los mismos jóvenes revisaban las grabaciones y eran autónomos en la toma de decisiones para repetir las escenas las veces que fuera necesario. Por otra parte, el registro del sonido en las tres piezas audiovisuales no fue el más óptimo, dado que los recursos con los que contaba el grupo investigativo no eran los más idóneos para el tipo de locaciones que, en su mayoría, fueron en exteriores. En cuanto a la edición de los videos, fue realizada por los facilitadores debido a los pocos recursos tecnológicos con los que contaba el grupo y el poco tiempo para realizar todas las etapas; no obstante, este aspecto se manejó con responsabilidad y tuvo como punto de partida el guion de trabajo elaborado por los estudiantes.

En total se elaboraron tres piezas audiovisuales que oscilan entre tres y cinco minutos, las cuales se analizaron desde el contenido y la estructura narrativa con la finalidad de reconocer su valor. Cabe agregar que la definición del mensaje principal y sus ideas secundarias fue de completa autoría de los participantes. Aunque cada uno de los grupos contó con el acompañamiento de los facilitadores, quienes ejecutaron los roles y funciones que requiere el logro del audiovisual fueron los jóvenes.

El primero de estos materiales, titulado "Pescando misterios", es una adaptación de un mito muy representativo del territorio, que se conoce como la "luz corredora". Algunos pescadores la describen como "una luz verdecita que corre por la ciénaga y de pronto se apaga", según Hipólito Cabarca, y para Elibanio Veleño "es un reflejo en el agua, una luz que se fue desapareciendo a medida que nos acercábamos".

Los jóvenes decidieron modificar este mito por la "luz corredora de basura", la cual describe a un monstruo que se alimenta de los residuos sólidos vertidos en la ciénaga y amenaza a las personas con llevárselas si no cambian sus hábitos. Así, los chicos le dieron una connotación de peligrosidad a la basura que es tirada a este cuerpo hídrico, ya que esta práctica, con el tiempo, puede causar graves afectaciones medioambientales. Asimismo, refleja una postura de preocupación por la falta de conciencia y responsabilidad de los habitantes que no contribuyen a la preservación de los recursos. Por esta razón, al final del video, los participantes comparten su preocupación por la falta de conciencia respecto al cuidado del medio ambiente y dan un mensaje para salvarse del monstruo de la basura: "Quiero contarles cómo librarse de la luz corredora de basura, teniendo los sitios de El Llanito limpios, lo que es la cancha, la iglesia, el colegio y sus casas", dice Brayan Pazos.

El segundo producto se llamó "Afectaciones de los sitios turísticos a causa de la contaminación". Este material recurrió al reportaje, género periodístico que involucra roles como periodistas o reporteros. Con un tinte más informativo, se enfocó en reconocer las transformaciones sufridas en sitios del territorio que antes gozaban de un buen cuidado, por lo cual eran frecuentados por turistas, pero que ahora han sido víctimas del abandono, el mal estado de la ciénaga y la inadecuada disposición de basura. Las tomas 
de apoyo registradas por ellos muestran el deterioro de lugares como el Típico, sitio que ofrecía antiguamente servicios a los visitantes. De igual manera, acudieron al uso de la entrevista para presentar el testimonio de un pescador que da sus aportes sobre la incidencia del no cuidado de los recursos naturales y cómo esto ha afectado al territorio en los aspectos cultural, económico y de salubridad.

A partir de esto, los participantes lograron indagar sobre posibles acciones que la comunidad podría llevar a la acción con el objetivo de propiciar el mejoramiento de aquellos lugares. Como cierre de este video, se identifica que los jóvenes proponen el reciclaje como una posible solución, ya que sería una forma de que aquellos residuos sólidos que son generados tanto por los habitantes como por los turistas fueran a un lugar adecuado y no terminaran en sitios como las orillas de la ciénaga, la cancha o el parque, espacios que fueron señalados por los estudiantes como principales depósitos de basura. Estas apreciaciones se obtuvieron en la primera parte de este proyecto, que consistió en el diagnóstico participativo.

La última pieza audiovisual narra desde una dramatización la historia de un adolescente muy apasionado por el fútbol, pero que no es muy consciente de la importancia de cuidar de los recursos naturales. Cuando el protagonista se hallaba bañándose en la ciénaga, es sorprendido por el espíritu de un hombre llamado Lucho Arango, pescador que fue asesinado por ser uno de los líderes ambientales más reconocidos de El Llanito. Este personaje trae un mensaje para el joven y le advierte que si los pobladores de ese lugar no dejan de tirar basura ocurrirá una catástrofe que afectará al corregimiento. Así pues, el chico se dirige donde su mamá para contarle el acontecimiento y juntos deciden dialogar con toda la comunidad para que sean las propias personas las que reflexionen sobre sus actos y puedan entender su responsabilidad de preservar las riquezas naturales que están a su alrededor.

En este audiovisual los jóvenes hacen referencia a Lucho Arango, una persona que trascendió en la región por su trabajo como defensor de la ciénaga, como se mencionó en la introducción de este artículo. Su representación en el video dota de simbolismo a la estructura narrativa y significa un acercamiento a la memoria de lo que ha sido la historia del corregimiento desde la perspectiva de las personas que han tomado las banderas del trabajo comunitario para defender, en este caso, la ciénaga y la pesca artesanal como una forma amable de explotación de los recursos naturales. Ahora bien, el mensaje de Lucho es claro y señala la necesidad de cambiar las costumbres de los habitantes de El Llanito, porque, al final, la ciénaga tiene un nuevo enemigo: los residuos sólidos, que en apariencia son inofensivos, pero, con el paso del tiempo y su acumulación en las aguas, terminan aportando al desequilibrio del ecosistema cenagoso.

Otro de los aspectos que se analizó fue el enfoque desde el cual decidieron construir la narración. Dos de ellos acudieron a la dramatización de historias a través de relatos 
ficticios y de la realidad, mientras que la otra pieza audiovisual se realizó desde el género periodístico del reportaje. Sin embargo, los tres abordajes transmiten las consecuencias que pueden desencadenarse cuando no existen acciones concretas para la preservación del medio ambiente. Además, se comprobó que el hilo conductor de los productos audiovisuales posee la estructura de inicio, nudo y desenlace, lo cual refleja la comprensión de los conocimientos que se compartieron con los participantes durante el taller teórico, en los que se destacó la importancia de la coherencia de las ideas en el lenguaje audiovisual con el fin de transmitir un mensaje que sea comprendido por las personas que vean los videos.

Finalmente, los audiovisuales participativos fueron socializados tanto con los participantes de la estrategia como con los docentes de la institución educativa San Marcos. Ellos destacaron de forma positiva en cada pieza las habilidades comunicativas de los estudiantes y se cercioraron de las capacidades que tienen. Esto fue significativo para los profesores, ya que vieron otras facetas de los alumnos que ellos no identificaban. Sin embargo, se pudo constatar con esta estrategia que, aunque los participantes de los talleres tenían un buen discurso sobre la importancia de cuidar el medio ambiente, sus prácticas cotidianas no reflejaban lo dicho. Asimismo, los adolescentes y jóvenes del proyecto, al ver los productos, se sintieron satisfechos con este primer acercamiento al lenguaje audiovisual, se conectaron con el resultado obtenido y fueron críticos con sus propios logros para reconocer que existen aspectos por mejorar, como sucede en todo proceso de aprendizaje.

\section{DISCUSIÓN}

Teniendo presente los aportes teóricos sobre el audiovisual participativo y los resultados obtenidos durante esta investigación, se constata que su uso sigue siendo significativo para estas estrategias educomunicativas, donde se valora el proceso como un espacio para el aprendizaje, el diálogo y la reflexión por parte de los participantes y especialmente de la comunidad. En este caso, se socializó con docentes de la institución educativa San Marcos, quienes reflexionaron sobre los aspectos más relevantes de los productos.

Los profesores, al observar las habilidades de sus estudiantes, lograron cambiar algunas percepciones, tal como señala Sandoval (2015), acerca de "generar materiales audiovisuales para la reflexión en contextos educativos formales y no formales, y porque, finalmente, con la realización de sus propios documentales, pueden despojarse de los estigmas, etiquetas y estereotipos" (p. 116). Esto puede entenderse como un primer acercamiento de relacionamiento entre adultos y jóvenes, que permite mayor libertad en el aula a la hora de proponer contenidos y espacios, lo que trae a colación los postulados de Freire y Kaplún sobre un modelo de educación horizontal que esté basado en el diálogo e intercambio de saberes, donde los estudiantes sean interlocutores de procesos pedagógicos que fortalezcan la participación. 
Desde esta lógica, no se puede reparar solo en las percepciones que genera la observación de los productos finales, pues hay riquezas e impactos propios en cada una de las personas que participaron en las diferentes etapas, que se escapan si solo se repara en la pieza lograda. Por lo anterior, es clave destacar en este punto que, si bien la herramienta del audiovisual genera un interés permanente en los adolescentes y jóvenes, un aspecto clave en el grupo con el que se trabajó fue que les costó organizarse, sobre todo en la etapa de preproducción, punto en el que deben establecer el rumbo y el enfoque del mensaje que quieren compartir con otros. Esto puede obedecer a características propias del contexto, la edad de los participantes o sus procesos de trabajo, en los que se prioriza la práctica, y sobre ella se van haciendo los ajustes que se consideren necesarios.

No obstante, y como un elemento transversal a todas las etapas de creación, cabe señalar que el uso de la herramienta audiovisual sí funcionó como un nuevo pretexto para que los estudiantes repiensen sus discursos sobre el medio ambiente. El hecho de perder su rol como receptores y empezar a crear sus propios contenidos abrió espacios de construcción colaborativa, de ahí que no resulte extraño que definir los mensajes en la preproducción haya sido una de las principales encrucijadas por las que atravesaron. La elaboración de un mensaje nuevo requiere poner en común acuerdo las ideas de varias personas, dotarlas de sentido y hacer que la narración que se escoja sensibilice al otro sobre el tema abordado. Una vez más se pone en evidencia que la comunicación, si bien no logra transformaciones inmediatas en las comunidades, sí propicia espacios para que las personas asuman roles activos y juzguen desde su realidad eso que les afecta, pero al mismo tiempo propongan soluciones. La comunicación termina siendo ese espacio en el que convergen las ideas y donde, a partir de las reflexiones críticas, se construyen nuevos mensajes que alimentan el tejido social de las comunidades.

Por otra parte, "el formato audiovisual constituye una evidente proyección para la difusión" (Rabadán, Bruzón y Montaño, 2015, p. 53) de situaciones o problemáticas que son identificadas por la comunidad, pero que no son una preocupación para ellos. Tal vez se deba a que no se sienten representados por aquello que ha formado parte de sus tradiciones, o también se remite a los cambios que ha sufrido El Llanito por la presencia de otras actividades económicas con las cuales obtienen más beneficios, con lo que dejan de lado su responsabilidad con la preservación y sostenibilidad de la ciénaga.

Este proceso del audiovisual también permitió que los participantes tuvieran un espacio de concertación y reflexión con el grupo sobre lo que deseaban comunicar y si realmente era importante contarlo, porque, aunque el tema fue propuesto desde la formulación del proyecto, ninguno de los jóvenes mostró o transmitió inconformidad sobre el tema medioambiental. Se resalta este aspecto con el fin de que se siga trabajando a favor de estrategias que ayuden a fortalecer más el accionar sobre el cuidado de los recursos, pues, como se mencionó anteriormente, los estudiantes tienen un amplio bagaje acerca del cuidado del entorno y sus recursos, pero son muy pocos los que son 
conscientes de que sus costumbres afectan de alguna manera al medio ambiente. Por ello, Mosangini (2010) reconoce que "la realización de videos documentales participativos es una herramienta de comunicación que sirve para analizar el mundo que nos rodea e implicarnos en su transformación" (p. 10).

Al ser este un primer piloto sobre la producción de audiovisuales participativos, se valoró en el ejercicio el trabajo de planeación de ideas, de organización y de ejecución de los roles. Además, se observó el liderazgo que tomaron algunos jóvenes de cada uno de los grupos para coordinar el plan de grabación. Sin embargo, se señala que el tiempo para el desarrollo de estos proyectos debe ser más extenso, con el objetivo de que profundice en espacios de reflexión con los participantes antes de actuar. Una de las dificultades de esta investigación para que los jóvenes llevaran a cabo los audiovisuales fueron los pocos días que tenían para hacer este ejercicio, al igual que otros factores externos como actividades programadas por la institución, que debían cumplir algunos de los estudiantes.

Finalmente, este proceso permitió fortalecer las competencias comunicativas de los adolescentes, en la medida que ellos mismos iban descubriendo aquellas habilidades que desconocían o que no habían tenido la oportunidad de explorar. Esto, de alguna manera, abre el camino para que sean los propios jóvenes los que propongan nuevas iniciativas que den cuenta de las necesidades o problemáticas que afectan a su comunidad. Además, el grupo de participantes de los talleres formativos cuenta con nuevos conocimientos acerca del papel de la comunicación, lo cual es significativo para toda la comunidad educativa, pues se podría llevar a cabo un proceso de retroalimentación con otros estudiantes que estén interesados en adquirir estas capacidades y saberes.

\section{REFERENCIAS}

Acosta, G., y Garcés, A. (2013). Colectivos de comunicación y apropiación de medios. Medellín: Universidad de Medellín.

Aguilera, C., y Polanco, G. (2013). Video comunitario, alternativo, popular... Apuntes para el desarrollodepolíticaspúblicasaudiovisuales. Recuperadodehttp://videocomunidad. univalle.edu.co/descargas/Libro_Video_Comunitario_Alternativo_Popular.pdf

Badillo, M. (2012). Propuestas de comunicación y educación ambiental a través del Facebook y el uso de narrativas digitales. Entramado, 8(1), 128-139. Recuperado de http://www.redalyc.org/pdf/2654/265424601009.pdf

Barreto, C., Bohórquez, H., y Piña, D. (2016). El empoderamiento de los líderes escolares: una estrategia para desarrollar la cultura ambiental en dos colegios públicos de Bogotá D. C. Tecné, Episteme y Didaxis (TED), número extraordinario, 599-605. doi: 2323-0126 
Bedoya, A., Moscoso, L., y Rendón, L. M. (2015). Incidencia de los procesos comunicativos en los proyectos ambientales escolares. Revista Lasallista de Investigación, 12(2), 75-83.

Bernal, C. (2016). Métodos cualitativos y cuantitativos en investigación científica. En Metodología de la investigación (pp.73-77). Bogotá: Pearson Educación.

Ceballos, J. (1999). El restablecimiento de las condiciones de habitabilidad: un proceso de y para la comunicación. En P. Duque (Comp.), Enfoques y metodologías sobre el hábitat. Memorias de una experiencia pedagógica (pp. 97-104). Medellín: Universidad Nacional de Colombia.

Ceccon, C. (2006). Escuelas comunicativas de calidad educativa y democrática. En R. M. Alfaro (Ed.), La otra brújula. Innovaciones en comunicación y desarrollo (pp. 255-258). Lima: Calandria.

Corrales, F., y Hernández, H. (2009). La comunicación alternativa en nuestros días: un acercamiento a los medios de la alternancia y la participación. Razón y Palabra, 70(14), 1-34.

Díaz, F. (2017). El neoextractivismo y el neodesarrollismo en los contextos latinoamericano y colombiano. Revista Controversia, 208, 57-98.

Espinosa, J. (2012). El video participativo: herramienta para la transformación social en procesos pedagógicos, sociales y políticos. Revista Nexus Comunicación, 1, 64-75. Recuperado de https://www.researchgate.net/publication/317926650_ El_video_participativo_herramienta_para_la_transformacion_social_en_ procesos_pedagogicos_sociales_y_politicos_Diseno_de_propuestas_para_ dos_casos_en_la_Peninsula_lberica

Freire, P. (1969). La educación como práctica de la libertad. Montevideo: Tierra Nueva.

Freire, P. (2004). Pedagogía de la autonomía: saberes necesarios para la práctica educativa. México D. F.: Siglo Veintiuno Editores.

Galeano, M. (2004). Estrategias de investigación social cualitativa. El giro en la mirada. Medellín : La Carreta Editores.

Gómez, R. (2013). Reflexiones sobre lo "alternativo" y la "alternatividad" en el campo de lacomunicación y la cultura en Argentina y América Latina. El quilibrista, 1(1). Recuperado de https://p3.usal.edu.ar/index.php/elequilibrista/article/ view/1354/1705

Gumucio, A. (2001). Haciendo olas: comunicación participativa para el cambio social. Nueva York: The Rockefeller Foundation. 
Kaplún, M. (1997). De medio y fines en comunicación. Chasqui, 58, 1-5.

Lunch, N. (2006). Una mirada al video participativo. Recuperado de https://issuu.com/ insightshare/docs/insights_into_participatory_video_-_722a87cb7e42bb

Martín-Barbero, J. (1987). De los medios a las mediaciones. Comunicación, cultura y hegemonía. Barcelona: Gustavo Gili.

Martín-Barbero, J. (2002). La educación desde la comunicación. Bogotá: Norma.

Mejía, E., Novoa, E., Ñaupas, H., y Villagómez, A. (2014). Metodología de la investigación cuantitativa-cualitativa y redacción de la tesis. Bogotá: Ediciones de la U.

Menéndez, Y., y Pedroso, W. (2017). Vinculación de adolescentes con la gestión ambiental en comunidades costeras de Cuba, a través de la educomunicación y el audiovisual participativo. Revista de Comunicación, 16(1), 147-165. Recuperado de https://revistadecomunicacion.com/article/view/1012

Mosangini, G. (2010). Documentales para la transformación. Guía para la elaboración de documentales sociales participativos. Recuperado de http://cajondeherramientas. com.ar/wp-content/uploads/2019/05/guia-documentales-socialesparticipativos.pdf

Orozco, G. (1994). Al rescate de los medios. Diseño democrático para los comunicadores. México D. F.: Universidad Iberoamericana.

Rabadán, Á. V., Bruzón, L., y Montaño, S. (2015). Identidad, cultura y desarrollo a través del audiovisual participativo: el caso de jóvenes del Proyecto Younth Path de la Unesco en Costa Rica. Alteridad, 10(1), 44-56.

Rocha, C. (2018). El audiovisual participativo y las ciudadanías de alta intensidad en Cazuca (Colombia). Revista Internacional de Comunicación y Desarrollo, 9, 42-56. doi: $10.15304 /$ ricd.2.9.5214

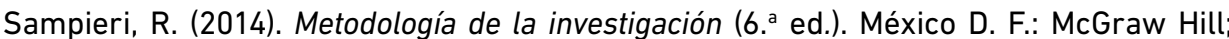
Interamericana Editores.

Sandoval, J. (2015). El documental participativo como proceso educativo: hacia la realización de proyectos audiovisuales con enfoque intercultural. Entreciencias, 3(6), 107-119. Recuperado de https://www.researchgate.net/publication/322590848_ El_documental_participativo_como_proceso_educativo_hacia_la_realizacion_ de_proyectos_audiovisuales_con_enfoque_intercultural 
